\title{
The Analysis of Click Bait in We Media Era
}

\author{
Zhi Zhou
}

\author{
University of California Davis, Davis, 95616 \\ Corresponding author. Email:hzizhou@ucdavis.edu
}

\begin{abstract}
In this era of information overload, all kinds of information come into our vision in different forms. Among them, click bait has been developed into a new advertising method at a remarkable speed. This paper briefly introduces the click bait in the form of "Yellow Journal" for the first time in history. Although the click bait can improve brand awareness and bring profits to businesses, the click bait is also consuming people's trust in the brand at the same time, and creative ideas will be exhausted one day. Based on the reasons above, the author analyzes the click bait and puts forward some suggestions on how to identify and avoid them. Through this study, the author finds that the clickbait has deeply penetrated into people's lives, so people should timely identify all kinds of clickbait to avoid potential scam.
\end{abstract}

Keywords: Click bait, Yellow Journalism, Advertising, Title

\section{INTRODUCTION}

Nowadays, in a rapidly developing society, time for browsing information has become more and more fragmented. In most cases, one glance at the title can determine whether audiences are interested in reading it. At this time, advertisers finds business opportunities, so the "clickbait" appears. Sometimes when browsing on the Internet, it is difficult not to be attracted by some exaggerated and bold words, such as "you WILL NOT believe!". After clicking into these titles, people will find out that they are actually advertisements. It is through this way that advertisers make money by cheating hits. The reason for the chosen topic is to study the deeper meaning for this communication phenomenon under the skin of the fast era. Based on this reason, the paper introduces the definition and background of clickbait. In addition, it focuses on the analysis of clickbait which discusses its influences in both positive and negative sides. What's more, the paper provides some advice about how to recognize clickbait from real news and avoid some potential possible scam. Through this analysis, this paper not only let the public know more about clickbait, but also help them identify different kinds of clickbait.

\section{THE INTRODUCTION TO CLICKBAIT}

Clickbait, as the name suggests, is a headline composed of some attractive and exaggerated words that does not match the content sometimes. In other words, it can also be understood as a deceptive advertisement in order to defraud more clicks through the first impression of audiences. People who create these baits usually take advantage of arousing the curiosity of the viewers, but do not provide enough information to get the viewers completely satisfied. In this way, they can increase their income by audiences' clicking on hyperlinks for more content [1].

Normally, the clickbait can be found in the results bar below when using a search engine which start with words such as "shocked" or "unbelievable" in bold. When the audiences click in and want to find out the whole article, the results always make them disappointed. Jianguo Deng mentioned in his paper, from a micro perspective, click bait can be understood as a writing style formed by advertisers to get more hits, which would make the audience feel cheated. From a macro perspective, click bait is a systematic productive mode to content producers [2] . In general, click bait, as a new communication phenomenon, is worthy of in-depth discussion and research.

\subsection{The Origin and Development of Clickbait}

History can know the rise and fall. Therefore, it is necessary to focus on the origin behind clickbait. In fact, the "Yellow Journalism" itself can be said to be the predecessor of the "click bait" nowadays. "Yellow Journalism" is based on sensationalism and describes a 
large number of intense news including crime, pornography, terror and other similar content, in order to increase the sales of newspapers. Yellow Journalism first appeared in the late 19th century because the editors of New York Journal and New York World scrambled to release some eye-catching and exaggerated news so as to compete for the "Yellow Kid" in the figure 1, which was a cartoon character that was popular in New York at that time. The Yellow Kid was created by Richard F. Outcault. The kid's name is Mickey Dugan. He is a bald, toothless and barefoot boy who always wears large yellow pajamas and strolls in a slum lane. The Yellow Kid always giggles. He uses an irregular and strange slang as his catchphrase. This slang is painted on his pajamas to satirize the billboard. And the name "Yellow Journalism" just comes from the news that was written to compete for the "Yellow Kid".

Today, a large number of "Yellow Journalism" are also popular on the Internet: in news websites, large amounts of click baits also use exaggerated sentences to attract attention, vulgar and useless; in pop-up news windows, bold headlines are obvious and superficial; even in YouTube video advertising, advertisers are no longer willing to spend time thinking about creative ideas. Instead, they flash a few big, meaningless words with the beat of music. In short, the purpose of advertising has changed from deepening the audience's impression to attracting the audience's attention. As Kuiken states in the paper, "Nowadays, a headline is often the primary way of getting a potential reader interested in an article" [3].

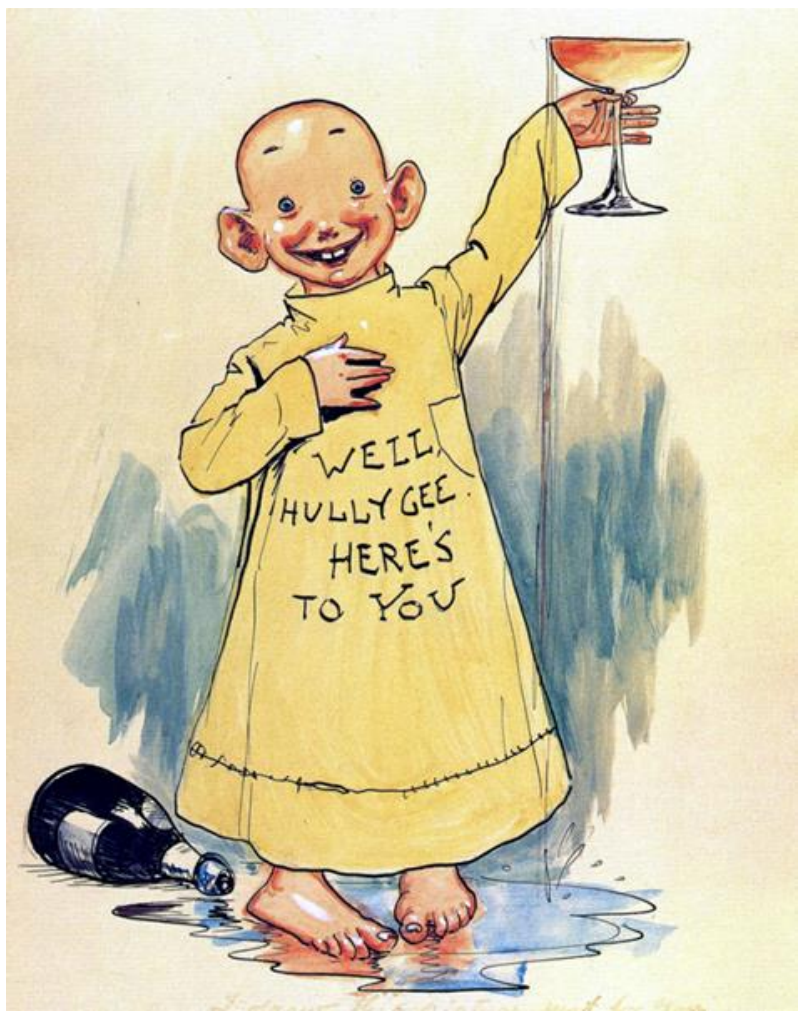

Figure 1 Yellow Kid

\subsection{Reasons for the Development of Clickbait}

Commercial interests drive the emergence and development of clickbait. Like traditional media, commercial interests are what every online media must pursue. Similar to traditional media, the profit of online media mainly comes from the sponsorship of advertising and the consumption of netizens. Both the investment of advertisers and the consumption of netizens mainly come from the authority and influence of the media. The authority and influence of print media mainly come from the sales of newspapers, while the authority and influence of TV media mainly come from the audience rating. Similar to the former two, the authority and influence of online media come from a new term: click rate. Click rate reflects the attention level of a content on the web page, so the click rate has also become the "lifeline" of online media. That is, the media is affected by the page views of articles, which depends on whether the click rate can be obtained and whether the click rate is high or not. The probability of an article being clicked is basically determined by whether the title is attractive. Therefore, page views are closely related to the title. Website needs page views, and a good title can bring a lot of attention and public exposure to the author. By some extent, the two are interdependent and mutually beneficial. The clickbait is doomed to the popularity due to the model of modern media. Thus, writing a thrilling title for the article is suitable for the current trend.

The clickbait became popular in such a short time because of its own characteristics. In addition to the importance of determining the audience's interest at a glance, the fast-spreading speed of clickbait cannot be ignored. While clickbait is popular, there are some articles lag far behind the clickbait articles in terms of communication speed and scope, even though they have knowledgeable contents. This can be attributed to a further reason: readers are credulous. With the development of science and technology, ways to access information are becoming more and more convenient. In this era when everyone can be the reader, the only thing that can directly explain the content of the article is the title. Readers identify the readability of different articles through titles of the articles unconsciously. For readers, the title has become the only standard to judge the quality of the article. Like TU Ling-Bo discusses in the paper, the scarcity of attention highlights the importance of time cost. Competing for attention is also competing for time [4]. In other words, click bait is the first important factor that will determine the attention of readers. Nowadays, the readers usually choose the news from the topic as the most concise way. Especially in the case of the same news event reported by different media, the readers will select the news that they pay most attention to from the topic: which topic can present the information most concerned by the audience; which 
topic can have the greatest influence and social impact. Therefore, the preference of the readers promotes the innovation of news media in headlines, thus promoting the emergence and development of clickbait.

The rapid development of the Internet provides the material basis for the emergence of clickbait. Nowadays, in the Internet era, a large number of news media emerge at the historic moment. People's computers and mobile phones are filled with massive information every day. Even if they are far away from these electronic devices, they will receive all kinds of information at any time. Therefore, how to choose what they really need or are interested in from such a huge amount of information has become the first problem that people have to face and solve. Therefore, some media put a lot of effort and energy in writing an attractive headline so that their news could be selected by readers in the numerous news. Especially in the mass of information, a "eye-catching" title is particularly important, so the clickbait is so produced and gradually developed.

Based on the above reasons, clickbait now has a growing trend and penetrates into people' lives.

\section{THE INFLUENCE OF CLICKBAIT}

However, how do people view the fact that clickbait has become popular? Every coin has two sides, people usually have two different attitudes toward clickbait. Some readers realize that the purpose of clickbait as a method is to cheat for audience's clicks to increase their income. While the information provided by clickbait itself cannot be of great help, and even cannot play any role for readers who would like to increase their knowledge by reading. This is the view of those who disagree with clickbait. In contrast, some people will think that clickbait is full of advertising creativity. The reason why they spread rapidly is benefited from their advertising strategy. No matter what people think of clickbait, it always imperceptibly affects people in both positive and negative ways.

\subsection{The Positive Influences of Clickbait}

For the increasing popularity of click bait, of course, it will bring some impact that cannot be ignored, whether for viewers or advertisers. First of all, from a good point of view, for advertisers, their purpose is to earn profit from advertisement. It is absolutely practical and understandable. In fact, the appearance of the click bait increases the income availability by viewer clicking on clickbait for more pageviews. As it is mentioned before, a perfect title is the key to an article. If advertisers want to get satisfactory profits, they have to work harder on the title. The title is the first and only thing that an article can show when people take their first glance and not even ready to click. Therefore, winning this first impression score is particularly important. Click bait should not only arouse the interest of people, but also attract them to click on the article to read. Now many website use algorithms for recommendation, once the audience gets more interested in the content, there will be more pageviews which leads to be recommended to more people as a high-quality source by the website. In this way, a beneficial cycle will be formed.

On the other hand, the impact of click bait also includes improving brand awareness. When people see the advertising articles of a brand for the first time, they will click in to view the content because of curiosity. The next time even if people no longer click, they will associate the brand with the keywords in the title. Over time, people will combine their first impression that the click bait brings with the brand name. If the advertisement is successful, these potential customers are always willing to give the brand a chance.

\subsection{The Negative Influences of Clickbait}

Although it is a good thing to see clickbait content perform well, there are some negative sides to be considered. From the perspective of marketing advisers, important events and hot-button issue can be the origin of creative idea of the click bait. The greater the creativity, the more intense the response may be. However, people do not make the same mistake twice. When the audiences laugh at the click bait at the first time, they will become numb and tired gradually. It can be predicted that if the trend of the click bait continues to be popular with this heat, people's daily lives will be occupied by the click bait all the time on websites, emails and TV. At that time, people's patience will be exhausted. Furthermore, the idea will run out. In other words, the idea can not always be fresh and interesting. For this reason, people's interest in click bait will not last forever.

Although there are some good ideas in terms of the perspective of advertising for click bait, the core is still the false advertisement that tries to deceive consumers with the first impression. Although it also expands the brand awareness, excessive click bait will lead to a decline in consumers' trust on the brand. When people click on the title but do not get the information they want to know, in the long run, the effect that the brand would like to achieve by customers clicking on the bait will be counterproductive.

Clickbait always uses exaggerated headlines to attract the attention of the audience, and it completely ignores the fact that news itself should follow the characteristics of objectivity, truth, fairness. Clickbait deviates from the truth, and willfully tampers with the original news facts, or even fabricates news out of nothing, which misleads the readers and causes them to have a wrong judgment on news events. 


\section{ADVICE ON IDENTIFYING CLICKBAIT}

To sum up, click bait is undoubtedly a strategy for advertising creators to improve click and bring benefits, but it is unworthy to risk losing brand reputation and exhausting creativity. Therefore, from the perspective of the audience, how to distinguish the click bait when seeing it? Following suggestions are provided for reference.

Firstly, if the question is asked in the title that can be answered directly, there is a certain chance that it is click bait. As Sugai Wang mentioned in the paper, Click bait often appears some interrogative or inverted sentences to form an atmosphere. Through this feature, the click bait can be identified directly [5]. For example, the title "What happened? I lost weight from $120 \mathrm{~kg}$ to $90 \mathrm{~kg}$ !" can be associated with fitness promotion or weight loss drug advertising. On the contrary, some titles, such as "what would students do if there was no college examination?" or "what am I thinking when I was 21?", which can cause people to think deeply and cannot get the exact answer at once, titles like these are reliable.

Furthermore, some titles that have no practical meaning may be click bait. For instance, "four things you must know", or "things will change your life", but articles ignore or even do not mention how to change in detail or what readers will get after reading this article. This kind of click bait arouses the curiosity of the reader, but usually readers do not get any useful information after clicking. Moreover, setting suspense in the title is also one kind of click bait. For example, "you will benefit a lot from losing weight like this" and "the one food no one should ever eat". The remaining key information was proposed to be released in the article, but the article with click bait did not mention related information. The last and most common one is that click bait articles usually use many exaggerated words, such as "shocked", "just now", "be careful". These words always appear at the beginning of the title to catch people's eyes, but again, they do not appear to have any practical meaning.

Enhancing citizens' rational thinking and correct judgment is an effective way to govern the phenomenon of clickbait. By helping the majority of the audience to distinguish different titles, and effectively guiding the younger or netizens to choose the right news, clickbait would have less potential readers so that it would gradually disappear. In addition, relevant departments should also fully mobilize the initiative and enthusiasm of netizens and encourage them to report, expose and crack down on clickbait, so that the media could grow in the supervision and criticism of netizens.

\section{CONCLUSION}

This article introduces what the click bait is and in what form it usually appears. Then it discusses the original form of the click bait which is "yellow journalism". It also explains why click bait is in line with the popular trend. In the past, the purpose of advertising was to impress people, but now with the development of technology, more and more fragmented time, advertisers begin to use click bait to try to attract viewers. Readers will also judge whether they are interested in clicking to read according to the title of the article. With different needs and understanding of advertising, click bait has gradually become a ubiquitous existence.

As Gothankar states in the article, "Clickbait has become a marketing tool in many sectors to entice users and thereby to generate revenue" [6]. As one of the biggest video websites in the world, YouTube, which people are most familiar with, generates hundreds of millions of clicks every day. Just imagine, if you subtract the number of clicks cheated by the click bait, how much will be left? In the era of information explosion, while facing a large amount of information, people have gradually lost the ability to select titles independently. Advertisers have added too many unrealistic words to the title in order to achieve the purpose of effective communication to the greatest extent and obtain more audiences. Time flies, and technology is advancing, the only thing you can control is your own choice.

\section{REFERENCES}

[1] D. S. Thakur and S. Kurhade,(2021) Context-based Clickbait identification using Deep Learning, pp. 1-5, DOI: 10.1109/ICCICT50803.2021.9510141.

[2] Deng Jianguo (2019). The Origin, Mechanism and Prevention of "Clickbait", News and Writing $8: 45-53$.

[3] Kuiken, Jeffrey, et al. (2017) Effective Headlines of Newspaper Articles in a Digital Environment, Digital Journalism 5(10):1300-1314.

[4] Ling-Bo, Tu (2017) The Origin, Influence and Controls of Clickbait Headlines in the Internet Era, Editorial Friend (4):44-48.

[5] Wang Sugai(2019). Analysis of the Clickbait's idiomatic linguistic devices, Journalism Lover 5:77-80.

[6] Gothankar, Ruchira \& Di Troia, Fabio \& Stamp, Mark. (2021). Clickbait Detection in YouTube Videos, pp.1-25. 